\title{
Geodesic fiber tracking in white matter using activation function
}

\author{
Temesgen Bihonegn ${ }^{\mathrm{a}}$, Sumit Kaushik ${ }^{\mathrm{a}, \mathrm{c}}$, Avinash Bansal ${ }^{\mathrm{a}}$, Lubomír Vojtíšek ${ }^{\mathrm{b}}$, Jan Slovák ${ }^{\mathrm{a}, *}$ \\ a Department of Mathematics and Statistics, Masaryk University, Czechia \\ ${ }^{\mathrm{b}}$ Brain and Mind Research Programme, Central European Institute of Technology, Masaryk University, Czechia \\ ${ }^{\mathrm{C}}$ Faculty of Electrical Engineering, Czech Technical University in Prague, Czech Republic, Czechia
}

\section{A R T I C L E I N F O}

\section{Article history:}

Received 28 July 2020

Accepted 9 July 2021

\section{Keywords:}

Diffusion Tensor Imaging

Ray-tracing

Metric tensor

Fiber tracking

Geodesic equations

\begin{abstract}
A B S T R A C T
Background and objective: The geodesic ray-tracing method has shown its effectiveness for the reconstruction of fibers in white matter structure. Based on reasonable metrics on the spaces of the diffusion tensors, it can provide multiple solutions and get robust to noise and curvatures of fibers. The choice of the metric on the spaces of diffusion tensors has a significant impact on the outcome of this method. Our objective is to suggest metrics and modifications of the algorithms leading to more satisfactory results in the construction of white matter tracts as geodesics.

Methods: Starting with the DTI modality, we propose to rescale the initially chosen metric on the space of diffusion tensors to increase the geodetic cost in the isotropic regions. This change should be conformal in order to preserve the angles between crossing fibers. We also suggest to enhance the methods to be more robust to noise and to employ the fourth order tensor data in order to handle the fiber crossings properly.

Results: We propose a way to choose the appropriate conformal class of metrics where the metric gets scaled according to tensor anisotropy. We use the logistic functions, which are commonly used in statistics as cumulative distribution functions. To prevent deviation of geodesics from the actual paths, we propose a hybrid ray-tracing approach. Furthermore, we suggest how to employ diagonal projections of 4th order tensors to perform fiber tracking in crossing regions.

Conclusions: The algorithms based on the newly suggested methods were succesfuly implemented, their performance was tested on both synthetic and real data, and compared to some of the previously known approaches.
\end{abstract}

(C) 2021 Elsevier B.V. All rights reserved.

\section{Introduction}

DTI (Diffusion Tensor Imaging) has become a clinical standard for studying and diagnosing neuro diseases. It is the non-invasive approach to obtain information on the neural architecture. Fiber tracking methods broadly comprise of two classes, probabilistic [13], and deterministic [4-8]. Probabilistic fiber tracking traverses all possible trajectories and provides a simulated distribution of the fiber tracts, which can be used in brain connectivity studies. Deterministic tractography methods are primarily based upon streamline algorithms where the local tract direction is defined by the principal eigenvector of the diffusion tensor. These approaches have been used to construct white matter anatomical connections in the human brain. In this work, we are considering the latter class.

\footnotetext{
* Corresponding author.

E-mail address: slovak@muni.cz (J. Slovák).
}

Earlier classical streamline based techniques [9] showed ineffectiveness in the reconstruction of highly curved fibers. Other difficulties with these methods appear in the isotropic (slightly anisotropic) regions where direction information is redundant. Apart from that, these methods are also sensitive to noise and fiber crossings.

To overcome the problems mentioned above [10-13] propose methods based on geodesics in Riemannian geometric space. These geodesics follow the shortest path locally between two points lying on the manifold. This path is optimal for the underlying actual fiber tracts. One class of such methods is based on Hamilton-Jacobi $(\mathrm{HJ})$ formalism. These methods are sensitive to local changes and provide a single solution.

In the works $[10,12]$ authors proposed to use the inverse of diffusion tensor as the metric tensor of the geometric space. Fuster and others [14] introduce modification of inverse metric, called adjugate tensor, which better explained Brownian motion on Riemannian space and overcame the issue with inverse diffusion tensor. 


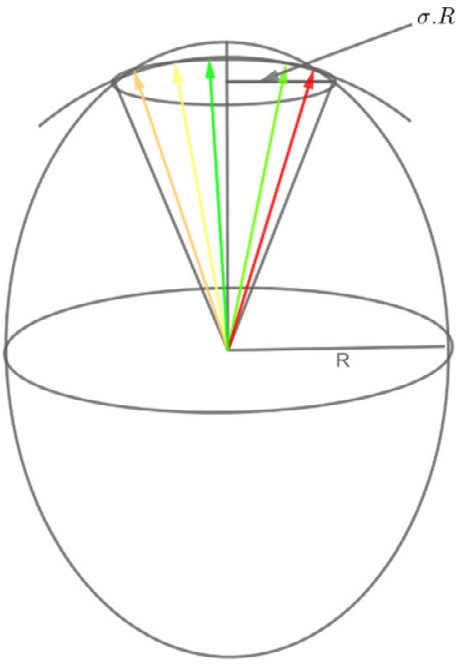

(a) elliptic cone

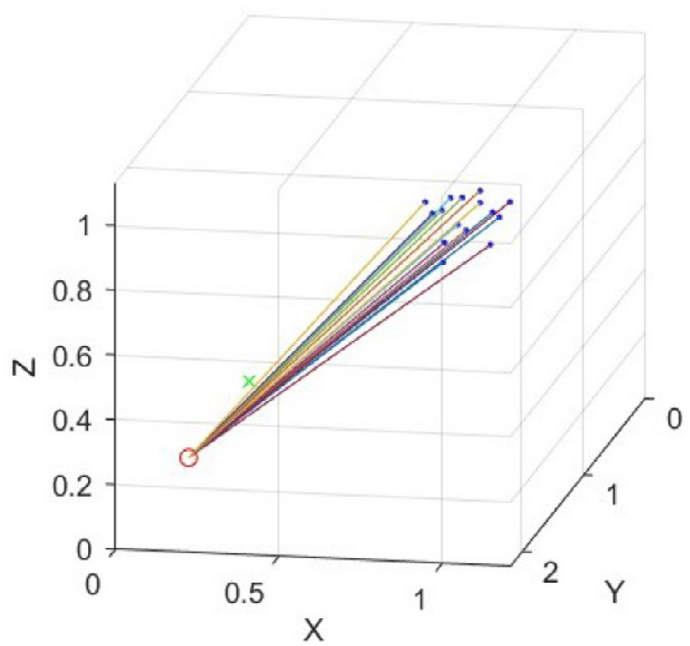

(b) cone shooting

Fig. 1. Initial shooting direction.

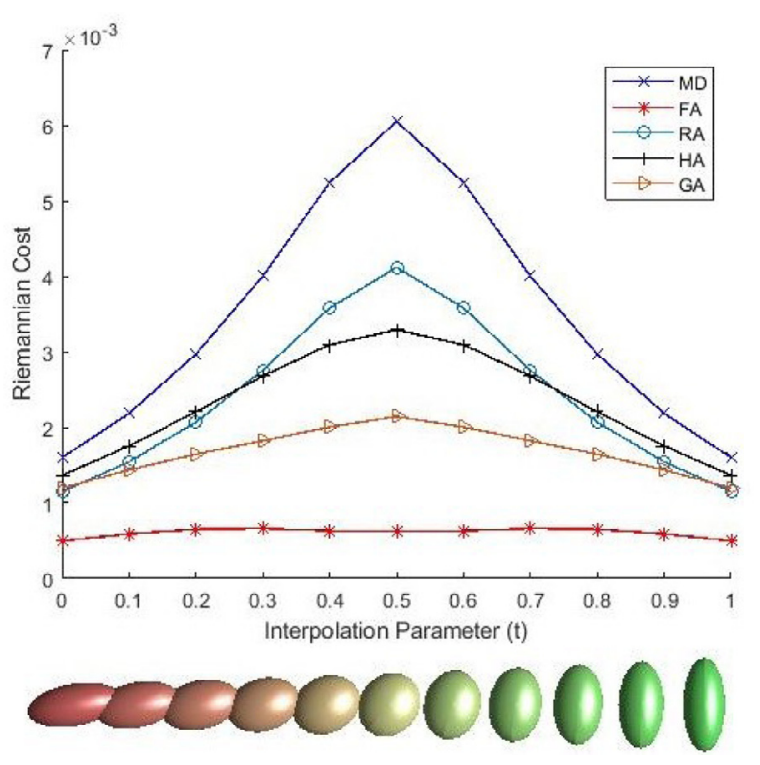

Fig. 2. The effect of interpolation between the tensors and the Riemannian cost from anisotropy to isotropic region. HA gives low Riemannian cost for anisotropic and high Riemannian cost for isotropic region.

In [15-17] Sepasian et al comes up with multi-valued raytracing method for anisotropic medium. Ray-tracing methods are based on the assumption that, locally in the medium, a wave or particle follows a path corresponding to the least action. Consequently, the directions of the path vary. These methods are capable of producing multiple geodesics between point and region in the medium.

Local variations of geodesics from underlying fibers are taken under consideration using Euler-Lagrange equations, but while traversing they deviate from the actual underlying path. The conformal rescaling or adaptive Riemannian metric is chosen for tractography in [18] and segmentation in their subsequent work [19]. Similar to their work, authors in [20] evaluated adjugate instead of $D^{-1}$ with or without sharpening. The choice of adjugate tensor as a metric does not resolve minimizing the Riemannian cost in all anisotropic or nearly anisotropic regions.

In this work, the contributions are as follows:[42]
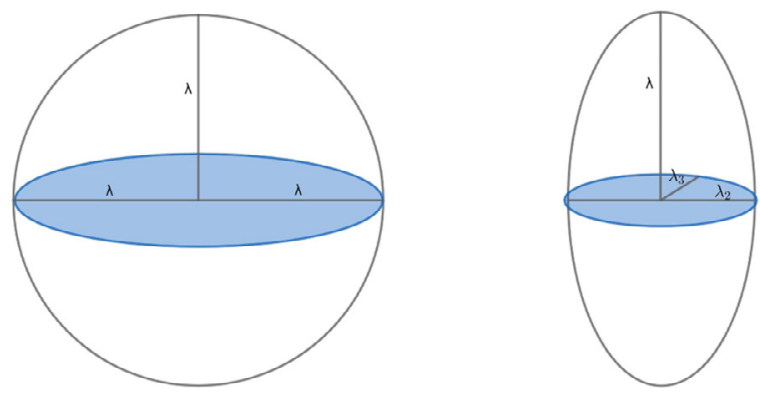

Fig. 3. An isotropic region (left) and anisotropic region (right).

1. Starting with the second order tensor data, we present a method to choose the appropriate conformal class of metrics where the metric gets scaled according to tensor anisotropy. We use the idea that the rotational information is related to the anisotropy of the tensor, and logistic function can be exploited to capture it. In particular, the rotational information is misleading in nearly isotropic regions in the presence of noise. The metric tensor is rescaled, according to this information. We compare various scalar anisotropies under the activation function.

2. Ray-tracing method deviates from the geodesics path in general. This problem is countered by feeding back the principal eigenvector direction of underlying interpolated tensor to ODE solver. This also enables the hybrid ray-tracing method to perform better in high curvature regions. We also enhance the method by local interpolation based on the so-called spectral quaternionic distance measures on the metric tensors.

3. We suggest to employ diagonal projection of 4th order tensors to perform fiber tracking in crossing regions. The diagonal components of the flattened 4th order tensor are second order tensors and lie in Riemannian space. We show that these components have potential to resolve fiber crossings even at small angle intersections.

This paper is organized as follows. In Section II, we review the geodesic-based fiber tracking approach. In Section III, parts A through $\mathrm{D}$, we introduce a modified ray-tracing method, which enables us to find multiple geodesics by shooting rays from point to region. We describe the use of activation function, which we call 


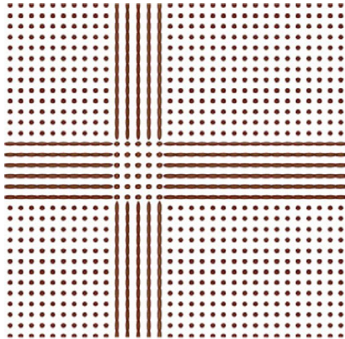

(a) case 1

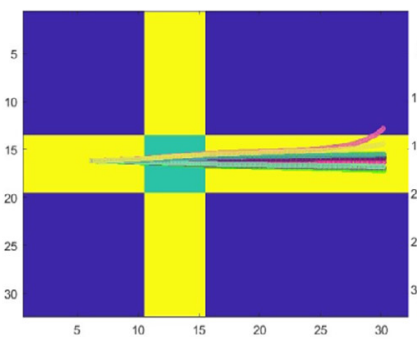

(b) $D^{-1}$

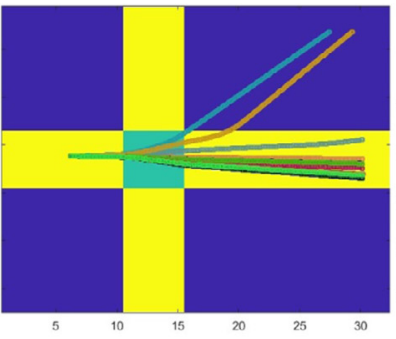

(c) Adjugate

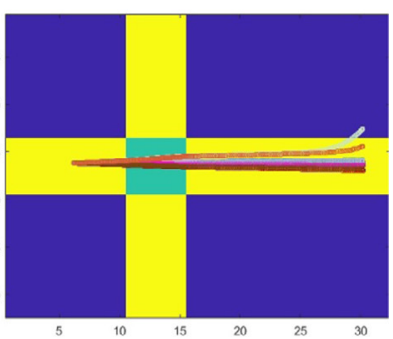

(d) $\beta$-scaled, $p=2$

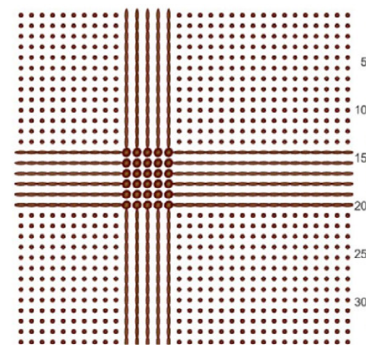

(e) case 2

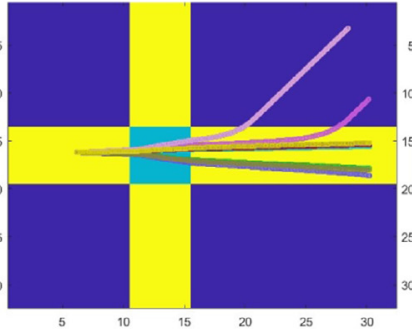

(f) $D^{-1}$

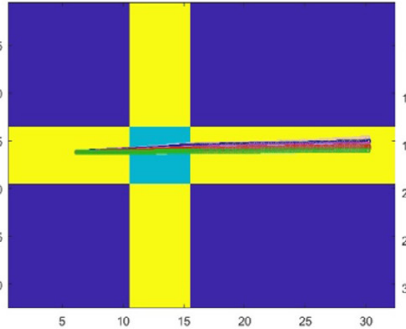

(g) Adjugate

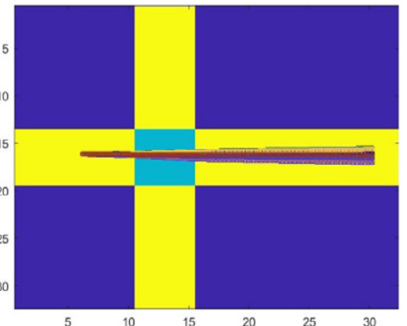

(h) $\beta$-scaled, $p=2$

Fig. 4. Comparison of the three metric tensors with the two extreme cases.
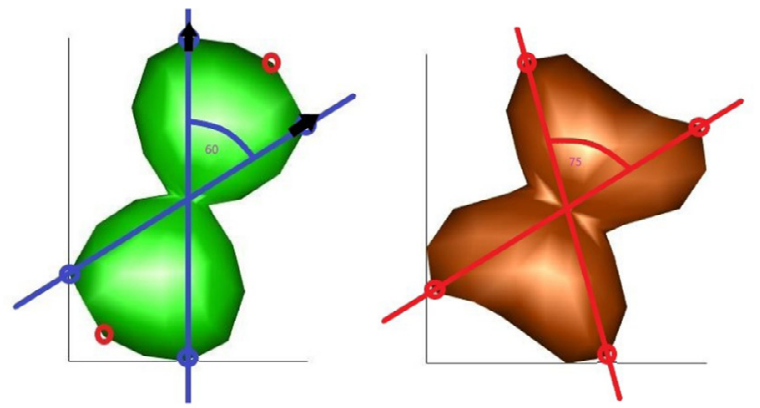

(a) angle between fibers $60^{\circ}$ (b) angle between fibers $75^{\circ}$

Fig. 5. 4th order ODF with angle differences between the two fibers.

$\beta$-scaled metric tensor. Next, we suggest how to employ the so called diagonal components of the fourth order tensors, [21], to resolve the fiber crossing even at small angles. Finally, in part $G$ we also comment on various choices of metrics suitable for local interpolation of tensor data. Section IV shows the results of our tracking approach on synthetic and real brain diffusion data.

\section{Background}

In geodesic ray-tracing, a small deviation of the geodesics from the direction of diffusion is preferred. It makes the geodesics robust to noise, but if this deviation is big, it needs a sharpening of diffusion tensor [15,22]. It can be done by powering the tensor. However, it causes artificial increase or decrease in volume, which is not required as the diffusion process is physical, and diffusion quality must be preserved. This is partially done by the normalization of the tensor. The sharpening strategy seems to result in better tractography. For more details, we refer the readers to [22].

The works $[23,24]$ explain the choice of inverse diffusion tensor as a metric in the context of DTI. It does not work for all cases. Another approach for modification of metric has been indicated by Hao [19], which has a similar effect as the adjugate metric proposed by Fuster [14]. The two approaches are build upon the conformal rescaling of the tensor. They use adjugate tensor with

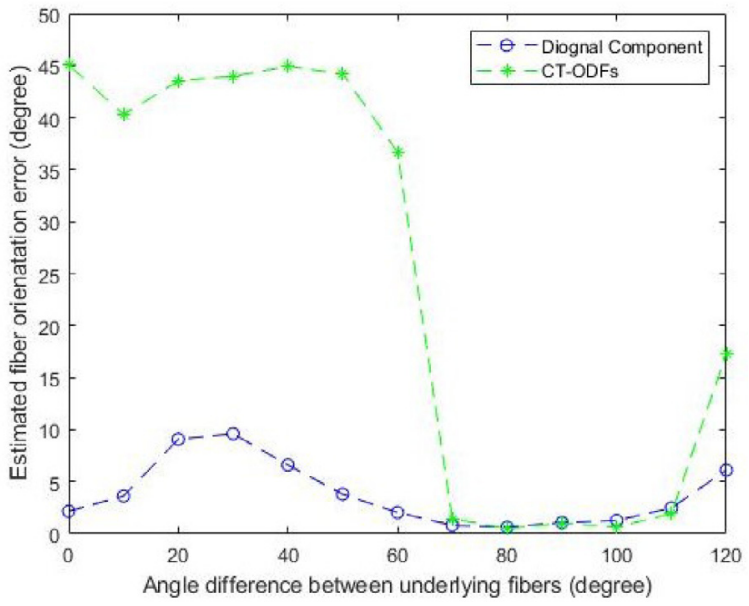

Fig. 6. Comparison of DC vs. CT-FOD.

sharpening to track high curvature fibers. The main idea to use inverse diffusion tensor as the metric tensor is to ensure that path is shorter if diffusion is stronger along the high anisotropic direction. This provides the minimization of the path, in essence, which can be treated as a geodesic. The ray-tracing method works under the assumption to consider a bundle of the rays together and provides a multi-valued solution. In this work, we consider a cone formed on the base of the ellipsoid.

Sepasian et al introduced a modified ray-tracing by adjusting the direction of geodesics based on computing Ricci curvature tensor from the metric tensor and its derivatives [25]. It provides a measure of the degree of deviation determined by the Riemannian metric tensor from Euclidean space. DTI model fails in the regions where fibers are merging, intersecting, and kissing. The second order tensor in DTI lies in the Riemannian space, which is well studied in [24,26-29].

The geodesic methods employing the Hamilton-Jacobi equation (HJ) fail in highly curved regions comparing to fast marching techniques. In [30], geodesics are considered as a function of posi- 


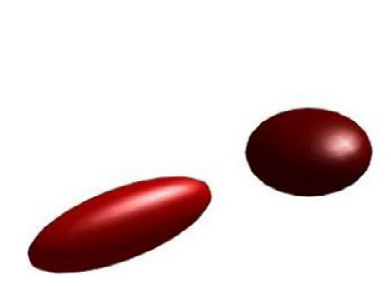

(a) $\log \mathrm{E}$

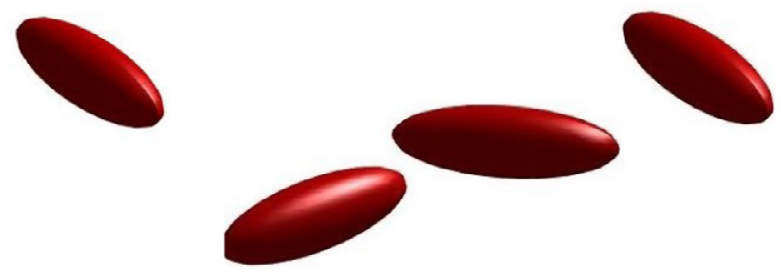

(b) SQ

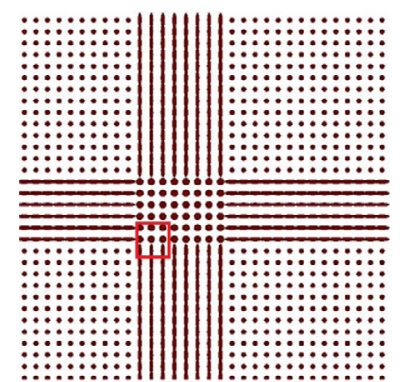

(c) Near the tensor at $(12,13)$ location

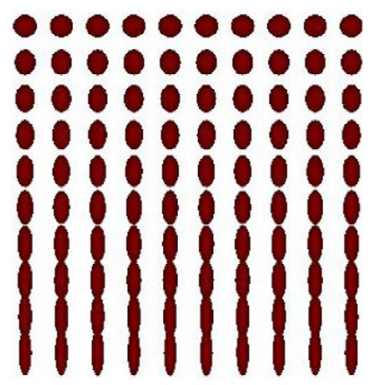

(d) LogE interpolation

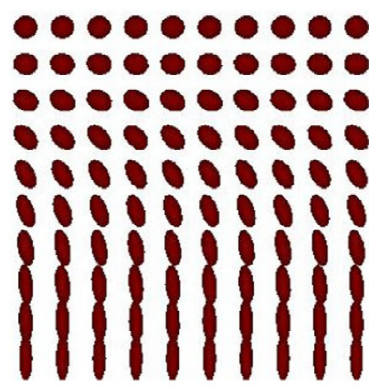

(e) SQ interpolation

Fig. 7. Local Interpolation of 2nd order tensors in crossing fibers using LogE and SQ interpolation.

tion and direction. In isotropic regions, the rays may deviate from the actual path $[22,31,32]$. Sharpening is helpful in these cases, as mentioned above.

During traversal, geodesic rays tend to deviate so that there is non-uniformity in their distribution across the regions, which causes less dense fibers. Ray density can be altered by changing the mesh size of interpolation. Anisotropic diffusion in Euclidean space is similar to the Brownian motion of water molecules in an isotropic medium in Riemannian Space [14].

\section{Ray-tracing via Activation function}

\subsection{Initial shooting direction}

Ray-tracing method is used to find the trajectory of the particle moving in medium. To compute the geodesics using this technique we need the initial position and direction. In DTI tractography, we can restrict the initial shooting directions. Let $R$ be the radius of the base and $\sigma \in(0,1)$, which adjusts the base of the cone. The different values of $\sigma$ provide different bases $(\sigma . R)$ of a cone. Directions are uniformly distributed over a spherical section of the cone, as shown in Fig. 1. This is done to restrict the shooting direction and to ensure the ray bundle remains densely packed.

The values of $\sigma$ approaching to 1 causes bigger perturbation. The more realistic way is to ray trace from point to region or region to region because it is not possible to know in advance if the initial and final points are connected [15].

\subsection{Activation function}

The logistic or activation functions are known for their common use in deep learning methods and statistics as cumulative distribution functions. One of their special cases is sigmoid functions, which are differentiable over real domain values and have positive derivatives at each point. To account for the tensors with negligible difference between the maximal $\left(\lambda_{\max }\right)$ and minimal $\left(\lambda_{\min }\right)$ eigenvalues, a smooth transition function is applied. In our case, we tested the following three functions with very similar perfor- mance:

$S_{1}(x)=\tanh (x)$

$S_{2}(x)=\frac{1}{1+\exp \left(-\frac{1}{2} x\right)}$

$S_{3}(x)=\frac{x}{\sqrt{1+x^{2}}}$

In our experiments discussed below, the function $S_{1}$ was used. Hilbert Anisotropy [34] is given by:

$H A=\log \left(\frac{\lambda_{\max }}{\lambda_{\min }}\right)$,

where $H A \geq 0, H A=0$ for fully isotropic tensor.

$\mathrm{HA}$ is a scalar measure of anisotropy and is scale-invariant (depends on the shape not the size of the tensor). It is also invariant to rotation and it is a dimensionless number reflecting microscopic diffusion at the level of tissues [33]. To choose an appropriate metric, we scale the Riemannian metric by an activation function, which is adapted according to the inherent anisotropy.

Let $\beta:=S_{i}(x)$ for $x=H A, i=1,2,3$, then the $\beta$-scaled metric is given by

$g_{\beta}=\beta^{-p} D^{-n}$,

where $D$ is the second order diffusion tensor $p \geq 1, n \geq 1$. In our experiments, we used $n=2$, and mostly $p=2$. Recall, $D$ belongs to the space $S^{+}(3)$ of positive definite $3 \times 3$ matrices (SPD). In particular, $D$ and all its powers are metric tensors.

Beside the Hilbert anisotropy, various scalar measures exist, which can serve as description for the degree of anisotropy of diffusion tensor. These measures can be composed with the above functions. They include: mean diffusivity (MD), fractional anisotropy (FA), relative anisotropy (RA) and geometric ones: geodesics anisotropy (GA), Hilbert anisotropy (HA) [33].

Spectral metrics allow for proper scaling of the rotational contribution according to the anisotropy. This is achieved by using the combination of the activation function with anisotropy scalar 


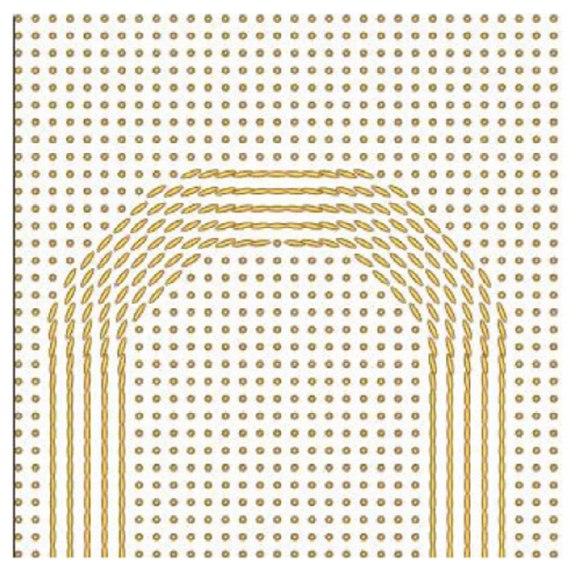

(a) Deep inverted U-shaped

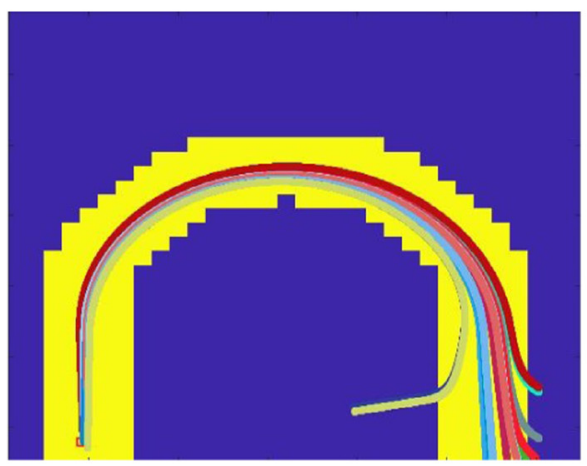

(b) adjugate

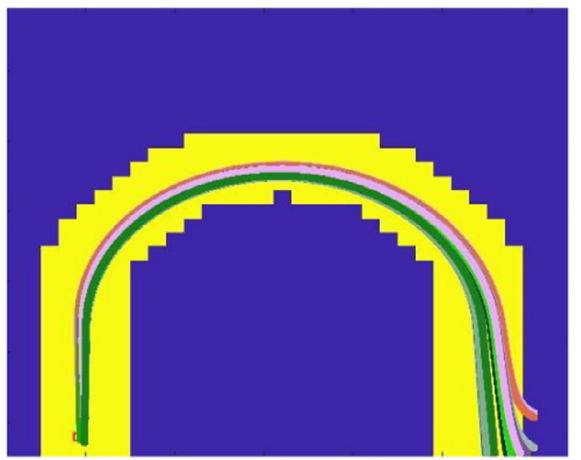

(d) $\beta$-scaled $p=4$

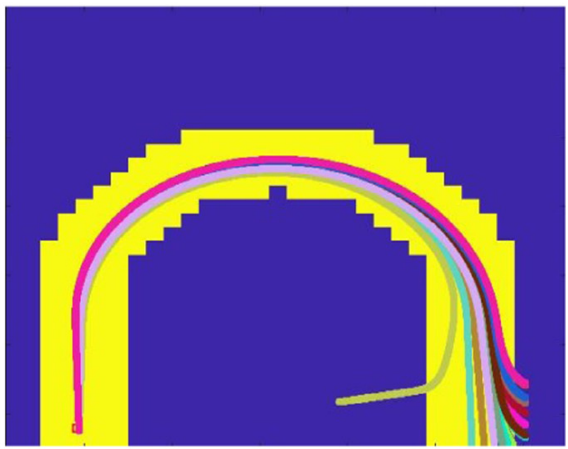

(c) $\beta$-scaled $p=2$

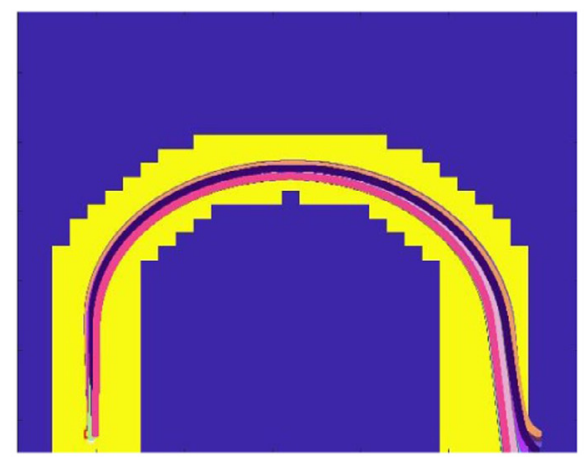

(e) $\beta$-scaled $p=6$

Fig. 8. FA images corresponding to (a),comparison of ray-tracing with ODE solver using adjugate, and $\beta$-scaled metric tensors from (b) - (e) respectively.

measure. In Fig. 2, we compare the Riemannian cost while considering these anisotropy measures under the activation function. From left to right the tensors exhibit high-low-high spectrum of anisotropies. The interpolation of tensors in between two extreme anisotropic tensors is obtained using Log-Euclidean metric shown in Eq. (11). Under this metric we can observe that the anisotropy is not preserved. There is variation in eigenvalues and rotational component of interpolated tensors.

The minimal Riemannian cost in anisotropic direction is given by $\beta \lambda_{\max }^{-2}$. It is observed that Riemannian cost increases as tensors achieve high isotropy in the middle of the spectrum and after which a smooth descend is noticed for HA case. The other scalar measures do not give linear interpolation as shown in [33], see Fig 3 there. HA is the only one of all above mentioned scalar anisotropy measure keeping affine combinations invariant.

\subsection{Governing equations}

The trajectory of a fiber pathway is computed iteratively from the hybrid approach, position from ODE solver, and direction equal to the principal eigenvector direction. The geodesic method in Riemannian manifold which is used to compute the trajectory of the fibers from ODEs is shown below.

Let $x(\tau)$ be a smooth and differentiable parametrized curve in the Riemannian manifold, $\tau=[0, T]$. The Riemannian length is given as follows:

$L(x, \dot{x})=\int_{0}^{T}\left(g_{\alpha \beta} \dot{x}^{\alpha} \dot{x}^{\beta}\right)^{1 / 2} d \tau$ 


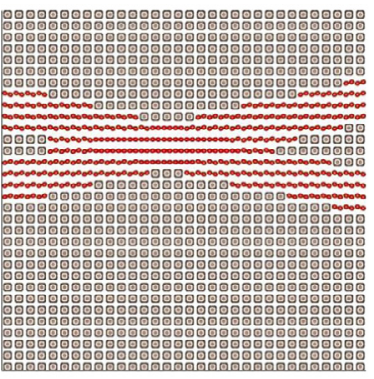

(a) 4th order tensor

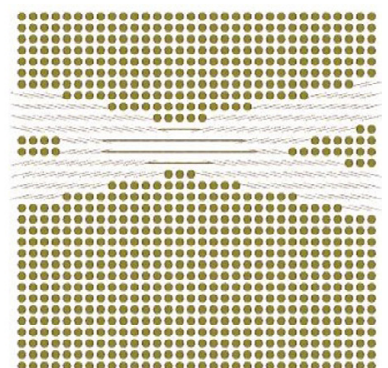

(b) Diagonal sum

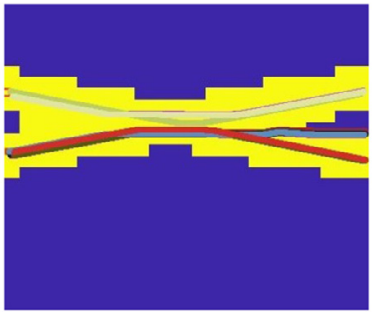

(c) $\beta$-scaled tracking

Fig. 9. Fiber tracing using $\beta$-scaled and DC when two fibers are close.

The geodesic is the curve that minimizes the length (4). The technique of the Euler-Lagrange equations for solving variational problems is explained, e.g., in [35].

Let $\dot{\chi}^{\gamma}$ and $\ddot{x}^{\gamma}$ be the first and second derivative with respect to $\tau$, respectively, of the geodesic for dimension $\gamma=1,2,3$. The geodesics are given by the following system of equations:

$\ddot{x}^{\gamma}+\sum_{\alpha=1}^{3} \sum_{\beta=1}^{3} \Gamma_{\alpha \beta}^{\gamma} \dot{x}^{\alpha} \dot{x}^{\beta}=0$,

where $\Gamma_{\alpha \beta}^{\gamma}$ are the so called Christoffel symbol, given by

$\Gamma_{\alpha \beta}^{\gamma}=\frac{1}{2} \sum_{\sigma=1}^{3} g^{\gamma \sigma}\left(\frac{\partial g_{\beta \sigma}}{\partial x^{\alpha}}+\frac{\partial g_{\alpha \sigma}}{\partial x^{\beta}}-\frac{\partial g_{\beta \alpha}}{\partial x^{\sigma}}\right)$

and $g_{\beta \sigma}$ denotes the matrix component of our rescaled metric, and $g^{\gamma \sigma}$ represents an element of its inverse. We compute the solution of Eq. (5) for the given initial position and multiple initial directions using the standard ODE solvers, such as fourth-order RungeKutta method. This gives us a set of geodesics connecting the given initial point, which we integrate until they hit the boundary. Depending on the Eqs. (5) and (6), we need nine symbols per dimension, for a total of 27 symbols. However, dealing with torsion free connections allows to exploit additional symmetries. The initial position is user-specified and directions are computed by forming a cone with a base of the radius.

The choice of power of $\beta$ is done experimentally. In our work, we have compared the results on synthetic data with $D^{-1}$, adjugate and $\beta$-scaled diffusion tensor. The experiment shows our approach works irrespective of configuration in terms of curvature and (an)isotropy of neighboring tensors.

Based on the observation that the ODE solver's output direction deviates from the actual fiber path, we used the principal eigenvector of the underlying interpolated tensor as input for the ODE solver. While picking up the principal vector direction, there are always possibilities of choosing two directions. At each iteration, we need to keep track of following the direction consistent with traversing fiber. This hybrid approach resulted in the traversal of geodesics in the high curvature cases and is robust to noise as well.

\subsection{Rescaling of metric tensor}

Illustration of Fig. 3 comes from [14] that advocate the use of adjugate diffusion tensor instead of the inverse of diffusion tensor as a metric tensor. The intuitive idea is to minimize the Riemannian cost along the trajectory.

Consider two tensors whose principal eigenvalues are equal. In $D^{-1}$ case, Riemannian cost (4) of (traveling along) an infinitesimal vertical line element scales by $1 / \lambda$. For adjugate case i.e., $d D^{-1}$, where $d=\operatorname{det}(D)$ the Riemannian cost for isotropic tensor is proportional to $\lambda^{2}$ (size of the shaded circle) and for anisotropic tensor it is $\lambda_{2} \lambda_{3}$ (proportional to the size of the shaded region). This method does not work if $\lambda>\lambda_{2}, \lambda_{3}$. When the area of the orthogonal cross section in the isotropic case becomes equal (i.e., same $\left.\lambda_{2}, \lambda_{3}\right)$ but their principal eigenvalues are different, adjugate tends to give the same Riemannian cost whereas our approach scales the metric appropriately according to the scalar anisotropy. The scaling coefficient takes zero value for isotropic and higher values for anisotropic cases.

HA is zero irrespective of the size of the isotropic tensor. This leads to same evaluation of Riemann costs for any isotropic tensor. Such scaling suggests that the diffusion of water molecules is uniform in all directions and hence the Riemann cost as well. In Fig. 4 two cases are depicted. Fig. 4(a) shows the case 1, where isotropic tensors in the intersection region are chosen with the smaller eigenvalues. Fig. 4 (e) shows the case 2 with larger eigenvalues. In both of the cases, the metrics $D^{-1}$ and adjugate induces different Riemann cost. However, in both cases, the $\beta$-scaled metric lowers the cost of traversing irrespective of the eigenvalues of isotropic tensors.

\subsection{Decomposition of 4 th order tensor}

Tuch [36] introduced the idea to use mono-exponential model for diffusion of water molecules in the tissues using multiple gradient directions:

$S=S_{0} \exp (-b D(g))$

For anisotropic diffusion this Eq. (7) is linear in the log domain, thus,

$\log (S)=\log \left(S_{0}\right)-b D(g)$

where,

$D(g)=\sum_{i_{1}=1}^{3} \sum_{i_{2}=1}^{3} \sum_{i_{3}=1}^{3} \cdots \sum_{i_{n}=1}^{3} D_{i_{1} j_{2} i_{3}, \ldots, i_{n}} g_{i_{1}} g_{i_{2}} g_{i_{3}}, \ldots, g_{i_{n}}$

Here, $D_{i_{1}, \ldots, i_{n}}$ are the coefficients of $n$-th order tensor, while $g_{i}$ are components of the unit gradient vector $g, b$ is the diffusion weighting factor, and $S$ and $S_{0}$ are drop in the signal in presence and absence of diffusion gradients respectively. Earlier methods based on the least square estimation do not ensure positive diffusion profile. The methods proposed in [37,38] ensures positive semidefiniteness of the tensors. We apply flattening of 4th order tensor, which gives $9 \times 9$ matrix, and eigen-tensors have the potential to reveal actual fiber directions [39]. The diagonal components approach [21] retains geometrical information of the full tensor. The diagonal component of this matrix is symmetric positive definite tensor [21]. In general, $n^{\text {th }}$ order tensor $T^{(n)}$ can be expressed as a matrix of $(n-2)$ order tensors:

$$
T^{(n)}=\left(\begin{array}{lll}
T_{x x}^{(n-2)} & T_{x y}^{(n-2)} & T_{x z}^{(n-2)} \\
T_{y x}^{(n-2)} & T_{y y}^{(n-2)} & T_{y z}^{(n-2)} \\
T_{z x}^{(n-2)} & T_{z y}^{(n-2)} & T_{z z}^{(n-2)}
\end{array}\right)
$$




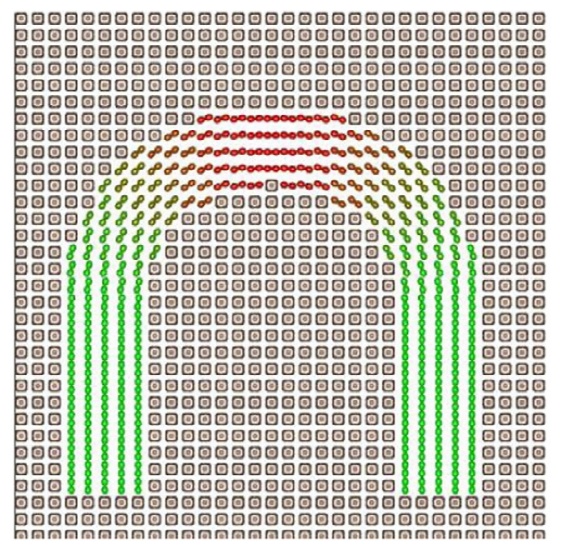

(a) 4th order inverted deep U shaped

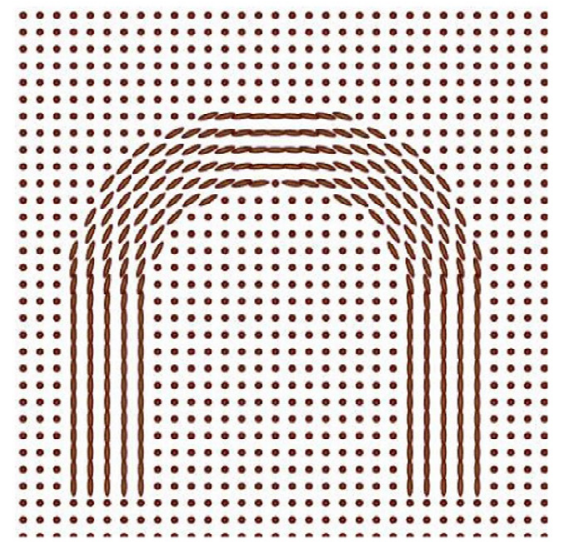

(c) Diagonal sum

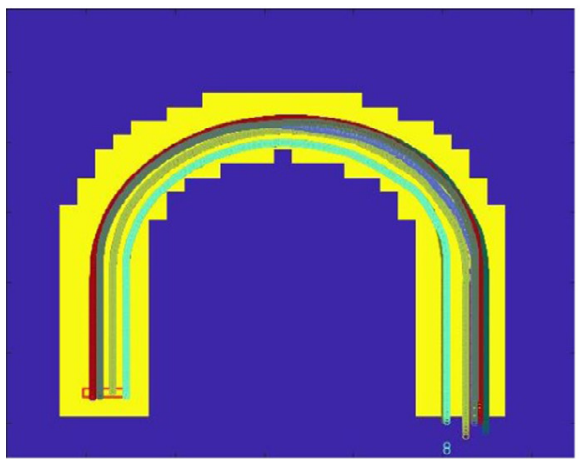

(e) $\beta$-scaled tracing

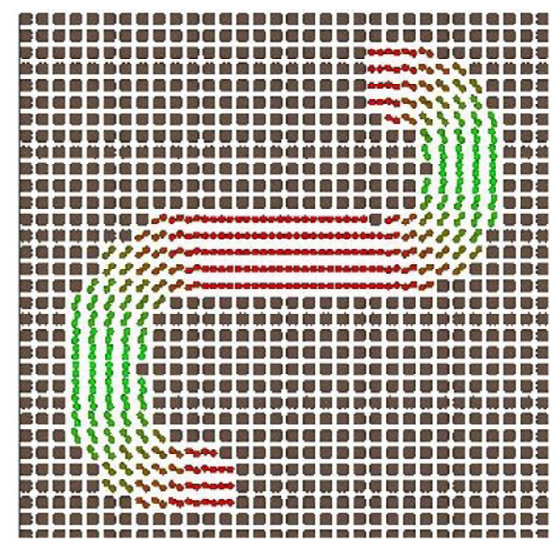

(b) 4th order in Reflected $\mathrm{S}$ shape

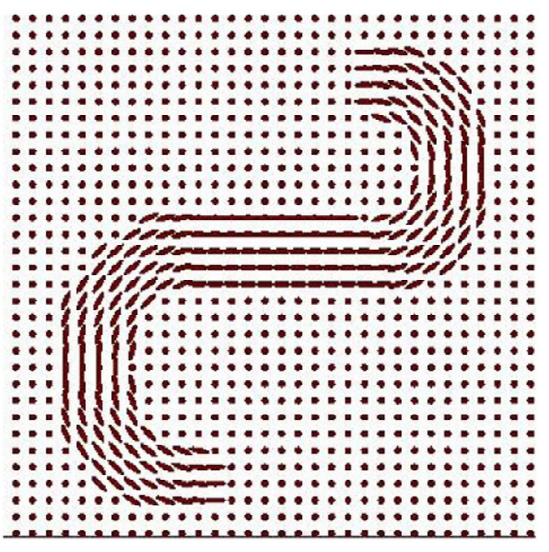

(d) Diagonal sum

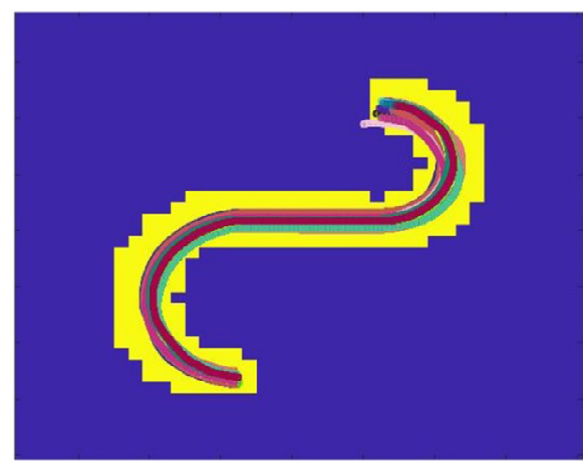

(f) $\beta$-scaled tracing

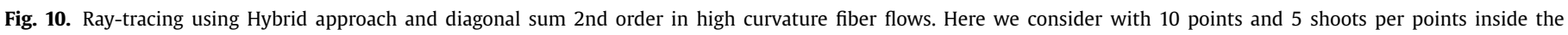
rectangular region.

For instance, the diagonal block element $T_{x x}^{(2)}$ in the fourth order diffusion tensor is given by

$\left(\begin{array}{lll}T_{x x(x x)} & T_{x x(x y)} & T_{x x(x z)} \\ T_{x x(x y)} & T_{x x(y y)} & T_{x x(y z)} \\ T_{x x(x z)} & T_{x x(y z)} & T_{x x(z z)}\end{array}\right)=\left(\begin{array}{lll}D_{x x x x} & D_{x x x y} & D_{x x x z} \\ D_{x x x y} & D_{x x y y} & D_{x x y z} \\ D_{x x x z} & D_{x x y z} & D_{x x z z}\end{array}\right)$

Another observation is that the flattening of 4th order tensor using diagonal components (DC) can potentially reveal the actual underlying fiber directions. This observation could be quite useful in fiber tracking.

\subsection{Resolution of fiber crossings at fine angles}

We have shown experimentally that these diagonal components produce small orientation errors in comparison to the Cartesian tensor fiber orientation distribution (CT-ODF) method [40] and [41]. In [40], Fig. 3, shows a comparison of orientation errors computed from CT-ODF method versus the other methods: QBI, DOT, MOVMF, and MOW. The other observation is about fuzziness in finding maxima using the CT-ODF method. These maxima provide the direction of underlying fibers. The maximal of ODF does not necessarily align with the actual underlying fiber direction. The CT- 


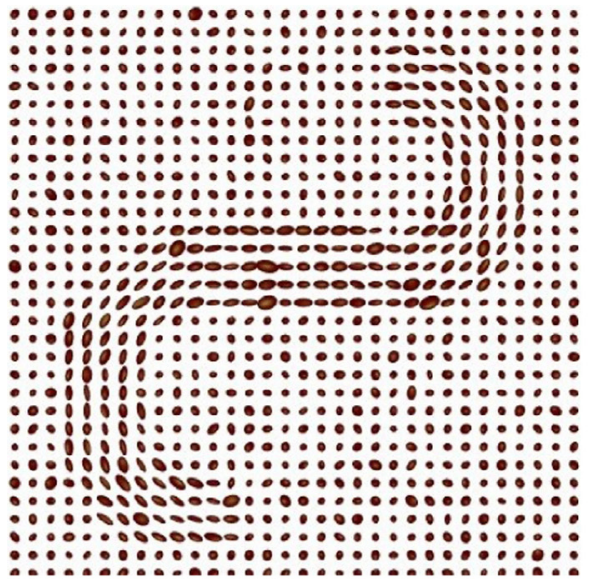

(a)

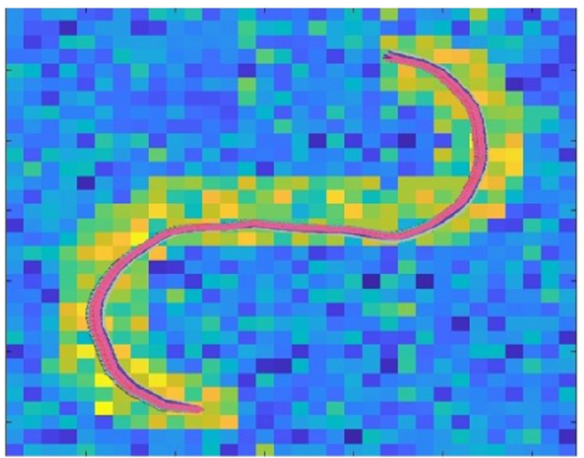

(c)

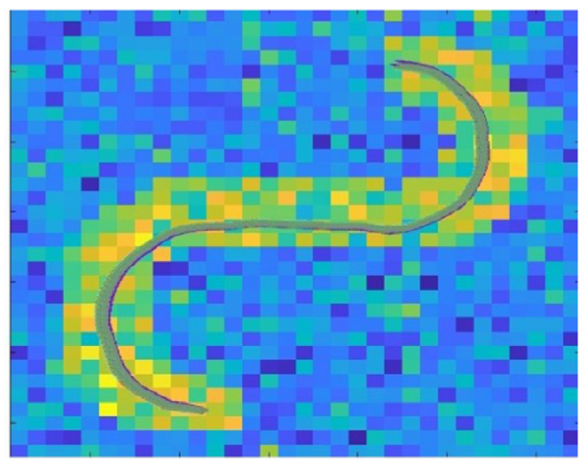

(e)

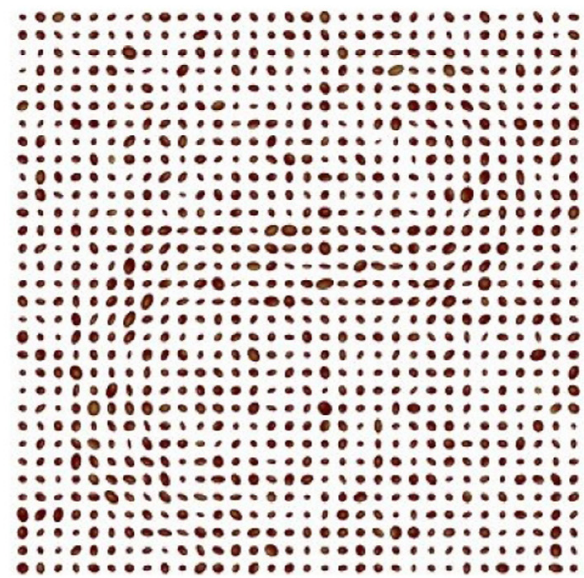

(b)

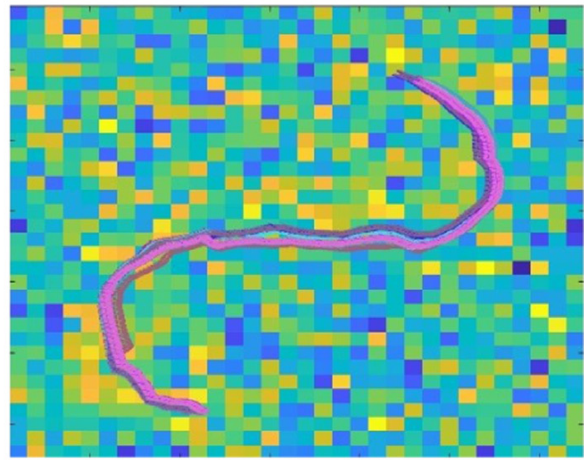

(d)

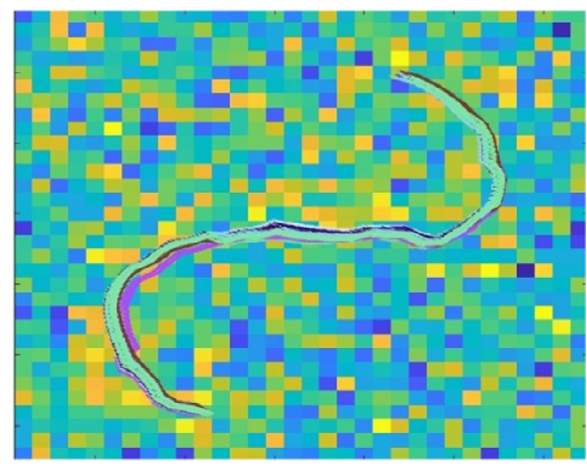

(f)

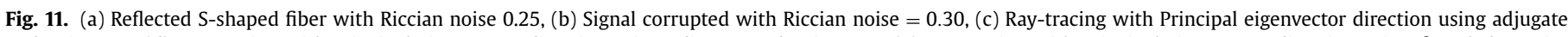

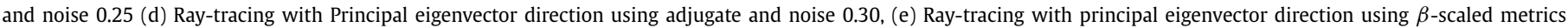
with $p=2$ and Riccian noise 0.25 , (f) Ray-tracing with principal eigenvector direction using $\beta$-scaled metrics with $p=2$ and Riccian noise 0.30 .

ODF method does the misalignment correction. The correction involves the computation of the maxima.

The CT-ODF method shows ambiguity in finding maxima, as shown in Fig. 5. In Fig. 5(a), when the angle between fibers is less than $70^{\circ}$, the points labeled by arrows are supposed to be the better choices for maxima than the middle one (point labelled by red colour). This effect disappears when the angle difference falls in $70 \leq \theta \leq 90$ range (see Fig. 5(b)).

Our projection to second order tensors mentioned in last section is devoid of this ambiguity. In Fig. 6, the known angle differences between the two fibers are shown on $x$-axis. These fibers are used to generate the ODFs. These angles are then retrieved us- ing CT-ODF and D-components. The resulting orientation errors are shown on $y$-axis. For the angle range $70 \leq \theta \leq 110$, performance of both the methods is comparable whereas for smaller angle differences there is a significant drop of the estimated error visible for D-components.

In the next subsection, we propose to use CT-ODF for reorientation and diagonal components for tracking fibers, particularly in crossing regions. This method is extendable to higherorder tensors; for instance, 6th order tensor has nine diagonal components which could resolve up to nine directions. However, practically more than 3 or 4 fibers per crossing seldom arise. 


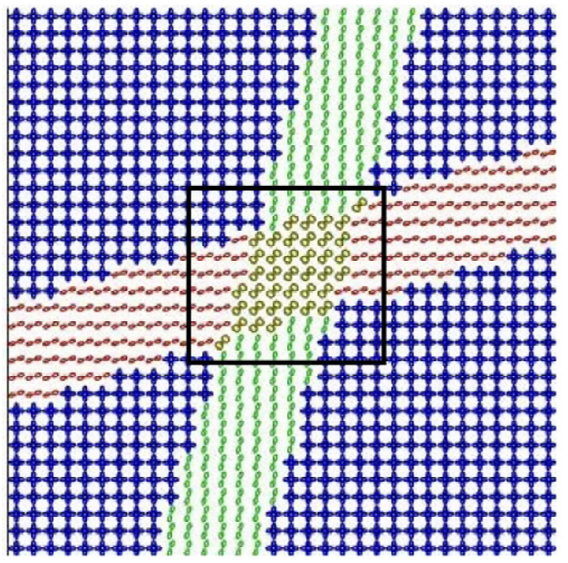

(a) 4th order tensor

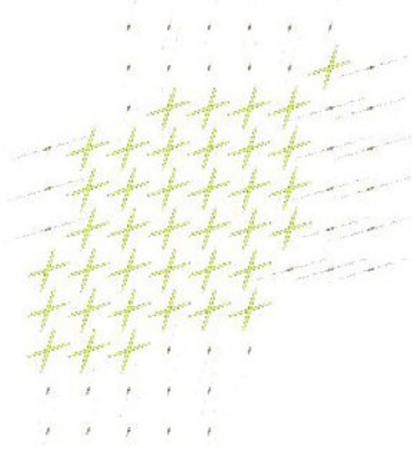

(c) DC of rectangular section

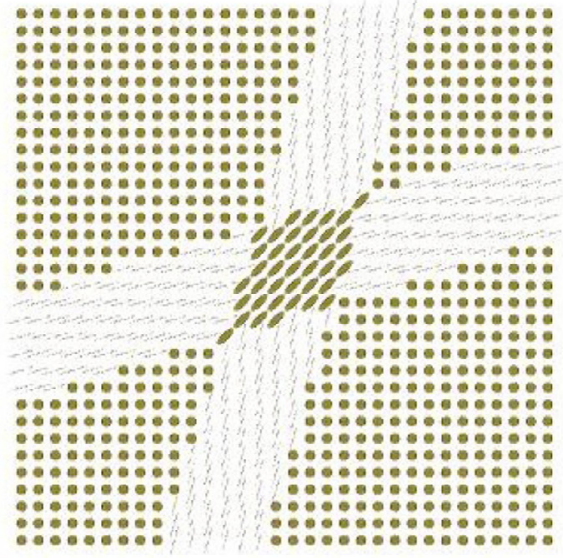

(b) Diagonal sum

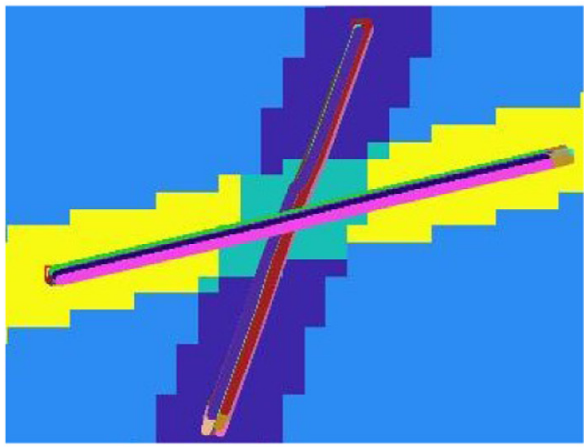

(d) Fibers using $\beta$-scaled

Fig. 12. Fibers computed using the hybrid approach and diagonal sum at crossing area.

Resolving crossing fibers. When dealing with the crossing regions, we enhance Algorithm 1 by working in two layers corresponding to two projections of the fourth order tensor to its diagonal components, see Algorithm 3.

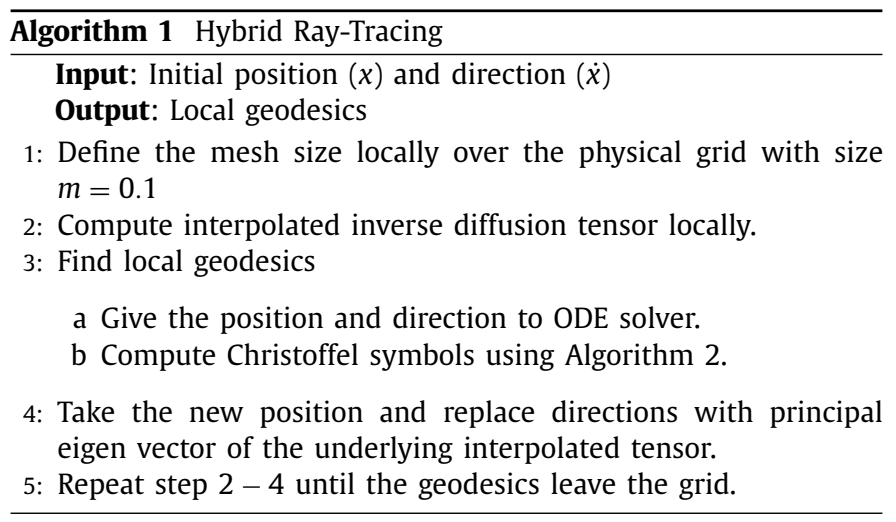

\subsection{Local interpolation effect}

The interpolation step in the algorithm affects the flow of geodesics while fiber tracking. Aside the elementary Euclidean interpolation of the tensor data, there are smarter choices available, including the Log-Euclidean (LogE), Spectral Quaternion (SQ), and spherical version of spectral quaternionic interpolation (SlerpSQ) [21], detailed explanation can be also found in [33].
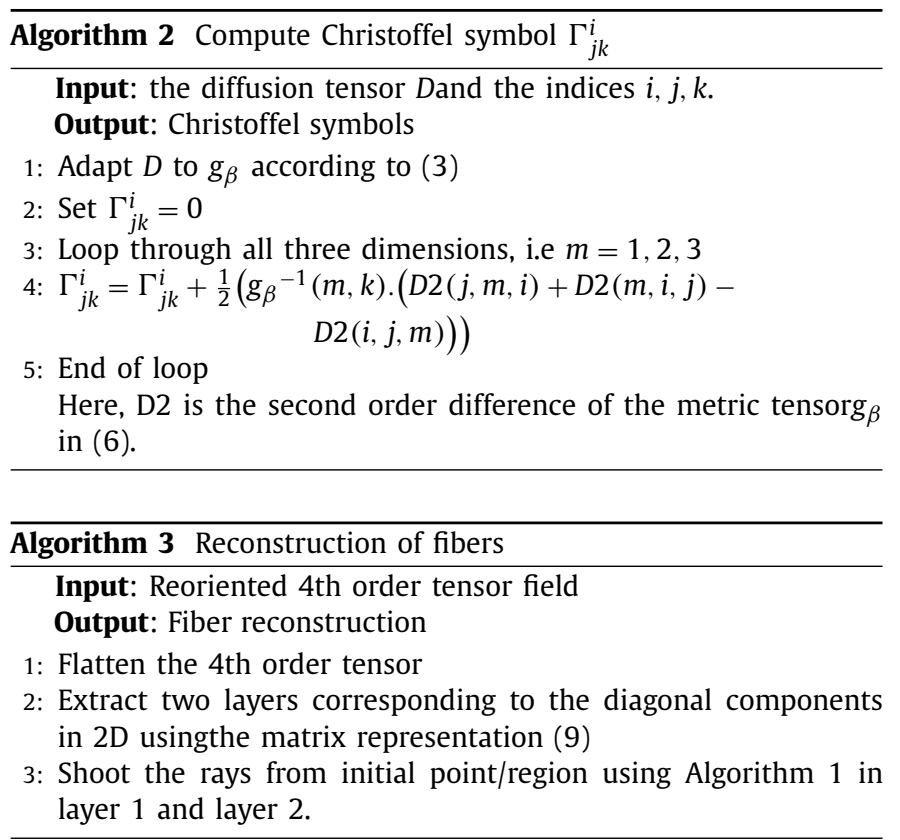

Log-Euclidean Interpolation: In this geometry, the distance between two tensors $T_{1}, T_{2} \in S^{+}(3)$ is given by

$d_{\log E}\left(\mathbf{T}_{1}, \mathbf{T}_{2}\right)=\left\|\log \left(T_{1}\right)-\log \left(T_{2}\right)\right\|$ 


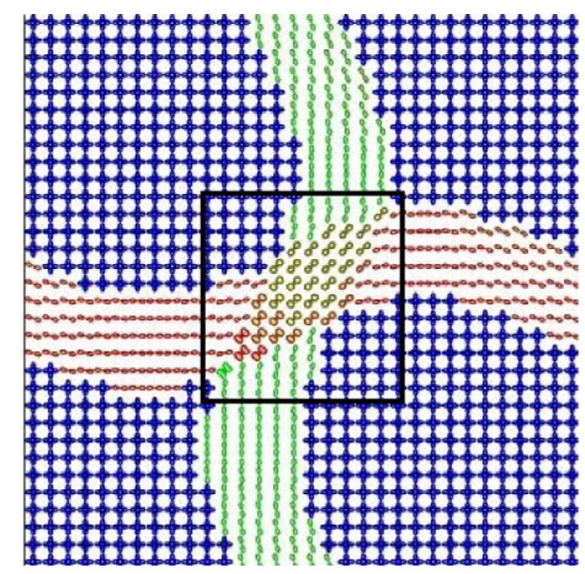

(a) 4 th order

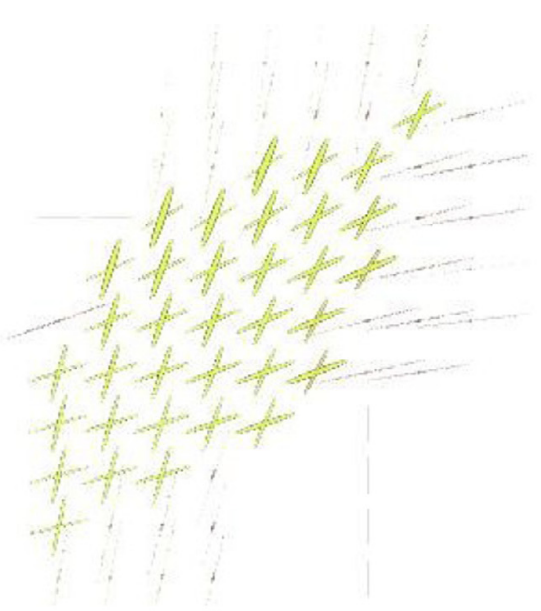

(b) zoom section

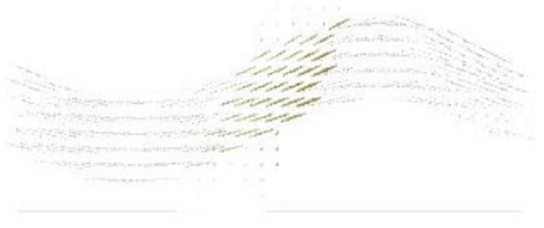

(c) first diagonal component (d) second diagonal component

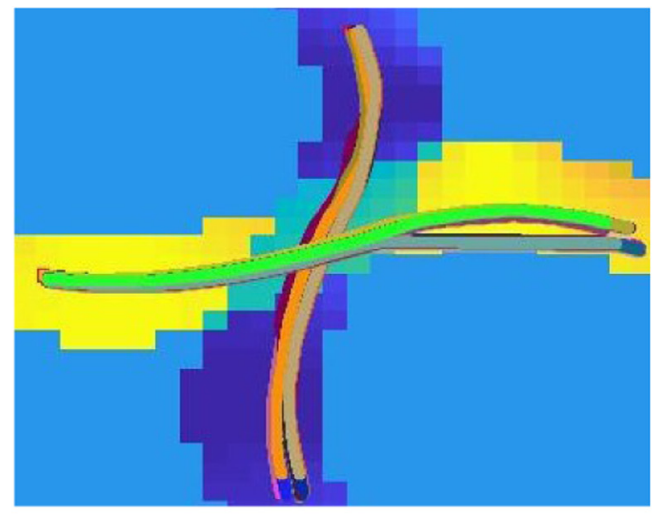

(e) $\beta$-scaled fiber tracking

Fig. 13. This figure illustrate $\beta$-scaled fiber tracking can trace in high curvature fiber flow using the hybrid approach and diagonal sum at crossing area.

It is based on the fact that the symmetric $3 \times 3$ matrices are diffeomorphic to $S^{+}(3)$ via the exponential mapping. The interpolation curve between two tensors is the geodesics curve $\gamma_{\text {LogE }}:[0,1] \longrightarrow$ $S^{+}(3)$, where the space $S^{+}(3)$ is a convex subset of the Euclidean space $R^{3 \times 3}$ of $3 \times 3$ matrices and it is given for all $0 \leq t \leq 1$ by:

$\gamma_{\log E}(t)=\exp \left(t \log \left(T_{1}\right)+(1-t) \log \left(T_{2}\right)\right)$
Spectral quaternion interpolation: The basic idea of spectral metric is to treat eigenvalues and eigenvectors of a SPD matrix separately. The eigenvalue decomposition of the SPD matrix in spectral geometry is $T=R \Lambda R^{T}$ into a rotation matrix $R \in \mathbb{S O}(3)$ and a diagonal matrix $\Lambda$ containing the eigenvalues, which provides a natural way of splitting the tensor. Thus using the spectral decomposition of a positive definite matrix, the interpolation curve is given by the 


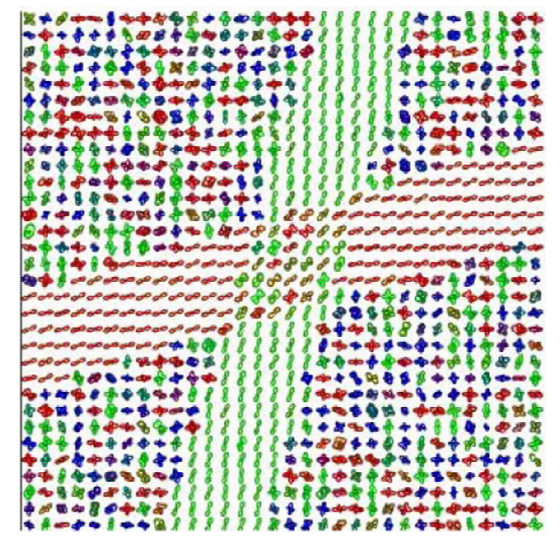

(a) Riccian noise 0.20

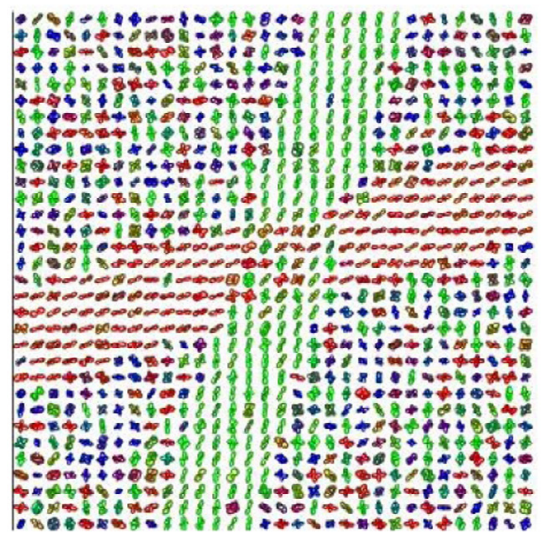

(c) 4th order with noise 0.30

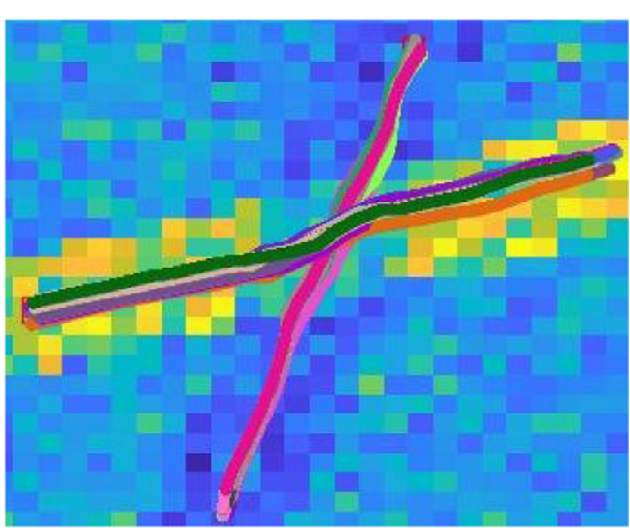

(b) $\beta$-scaled tracking with noise 0.20

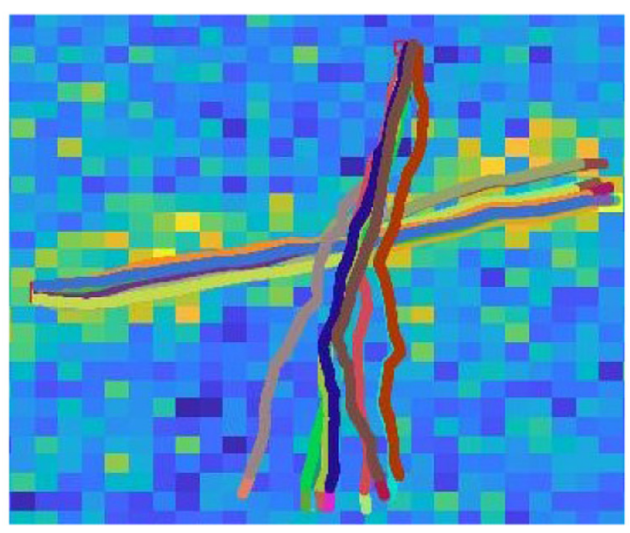

(d) $\beta$-scaled tracking with noise 0.30

Fig. 14. Fiber tracking in crossing fibers under noise.

equations

$S(t)=R(t) \Lambda(t) R(t)^{T}$,

$R(t)=R_{1} \exp \left(t \log \left(R_{1}^{T} R_{2}\right)\right)$

$\Lambda(t)=R_{1} \exp \left(t \log \left(R_{1}^{T} R_{2}\right)\right)$

The geometric interpretation of the interpolation curve is a geodesic in the product space of the Lie group defined as $G=$ $\mathrm{SO}(3) \times \mathrm{D}^{+}(3)$, where $\mathrm{D}^{+}(3)$ is the group of diagonal matrices with positive elements. In [21], both SQ and SlerpSQ have a similar effect in interpolation, we choose SQ interpolation for fiber tracking.

Fig. 7 (a) and (b) show the spectral metric preserves anisotropy, which is crucial in fiber tracking application. In Fig. 7(c), a tensor located at $(12,13)$, the rectangular section is considered. The tensor at this position is part of the vertical fiber and is underlying to the uniform background and not cross-section with horizontal fiber. Fig. 7(d) is interpolation in its neighborhood using the LogE metric and Fig. 7(e) does the same with respect to the SQ metric. The flow of interpolated tensors is more accurately captured in Fig. 7(e), which shows interpolation flows towards the left. Spectral metrics are known for robustness with respect to noise, in segmentation of curved fibers, and presentation of anisotropy.

\section{Experiments and results}

\subsection{Results on synthetic data}

For the experiments, we generate synthetic tensor fields with many configurations that have similar properties to many white matter tracts in the brain. The synthetic images are simulated using a signal generated with b-value $1500 \mathrm{~s} / \mathrm{mm}^{2}$ with a signal without gradient impulse $S_{0}=1$. Total of 81 gradient directions are chosen, which are uniformly distributed over the sphere. We use the adaptive kernel method to create fibers as detailed in [42].

In Fig. 8, deep inverted U-shape is considered with four points in the starting region and five shooting per point. It is visible that the adjugate metric fails to trace the fibers as it approaches the target region. $\beta$-scaled metric tensor geodesics follow the fibers well, and higher power of $p$ produces smooth fibers and increases fiber density. Fig. 9 shows tractography result on the layer of the diagonal components where the two fibers cross closely (cf. Fig. 5(a)).

Fig. 10 shows that the hybrid approach can trace in high curvature fiber flows. On top of that, the 4th order tensor field image is shown with 2nd order tensor field obtained by sum of the diagonal components. This produces sharp images contrary to DTI.

In Algorithm 1, the ODE solver method increases the deviation of geodesic along the path. To overcome this problem, we feedback the principal eigenvector direction of the underlying interpolated tensor to ODE solver. This causes geodesic deviation to disappear and leads to better performance under the three metrics. We tested our method on different high curvature fiber flows, as shown in Fig. 10. 


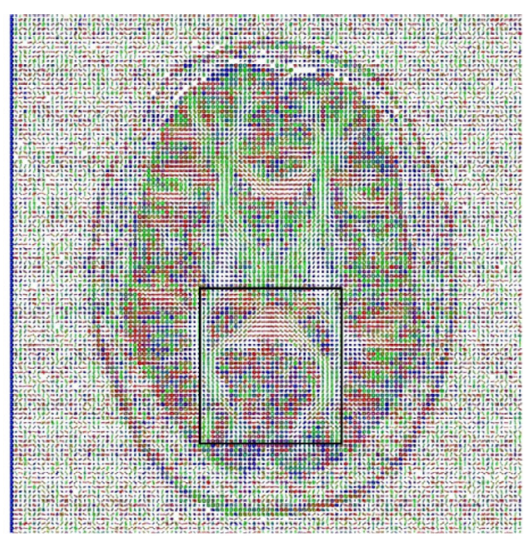

(a)

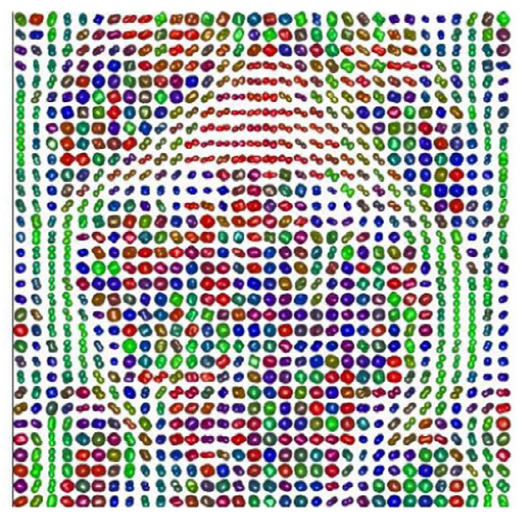

(b)

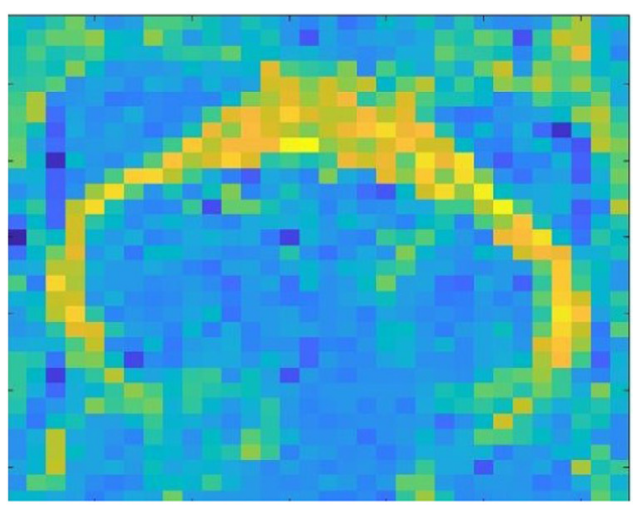

(c)

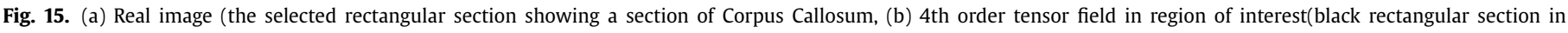
Fig (a)), (c) Fractional Anisotropy real Image.

Fig. 11 shows the S-shaped configuration corrupted with Riccian noise. The hybrid method acts robust and stable even in case where the fiber is poorly visible Fig. 11(b). We show results of geodesic tracking on synthetic data for crossing fibers based on $\beta$ scaled metric and diagonal component approach.

Fig. 12 depicts two linear fibers intersecting at small angle. Fig. 12(c) shows the two components in the intersection region. These components are sharp and follows the trajectory of their individual single fibers. Fig. 12(d) shows the $\beta$-scaled metric tensor used for tracing the fiber bundle.

Fig. 13 has two curved fibers intersecting. The Fig. 13(b) shows the diagonal components in the intersection region. Fig. 13(c) represent horizontally orientated regions of fiber curves whereas 13(d) indicates the vertically oriented regions and 13(e) is the result of $\beta$-scaled metric tensor tracking. In Fig. 13(c and d), the diagonal components are able to align along with the correct running curved fibers.

In Fig. 14, the image shows two linear fibers crossing at sharp angles corrupted with noise. In this difficult case our method is able to reconstruct the fibers and behaves robust.

\subsection{Results on real data}

Finally, we shortly comment on the results of our tracking algorithm applied to real images of the human brain.

The DW-MRI image consists of total size $114 \times 114 \times 70$ and each voxel is the size of $2 \times 2 \times 2 \mathrm{~mm}^{3}$. The real images are obtained by applying gradient in 64 diffusion directions with diffusion weighting factor $b=1500 \mathrm{~s} / \mathrm{mm}^{2}$ with single reference image $(b=0)$. We have used generalized logistic function (1a) as activation to test on real images.

In Fig. 15, a rectangular section of the Dorsal Longitudinal Fasciculus (DLF) is selected. The Fig. 15(c) is corresponding to FA scalar image, while (b) depicts the 4th order tensor.

In Fig. 16, 10 points were randomly picked in rectangular section on the top of the structure. Five geodesics are shot per point in both directions. The results indicate that most of the fibers trace the white matter structure in all three cases. Results under $\beta$ scaled metric tensor produce smoother geodesics.

The fiber crossing resolution method proposed above was not used in this experiment.

\section{Discussion}

We propose a new geodesic based tractography method by using a $\beta$-scaled metric tensor. This metric tensor is adapted according to the inherent anisotropy property. We have shown that the performance of adapted metrics by means of sigmoid function as activation function composed with the Hilbert anisotropy is better than performance of the classical metric and it also performs better than adjugate metric for highly curved fiber flows.

To increase the accuracy of the tracking approach, we iterate local geodesics tracing via Runge-Kutta ODE solver in the interpolated grid of tensor data, initiated by the principal eigenvector direction, called the hybrid approach.

Further, we propose to exploit the potential of using the diagonal components of 4 th order tensors, in particular for capturing 


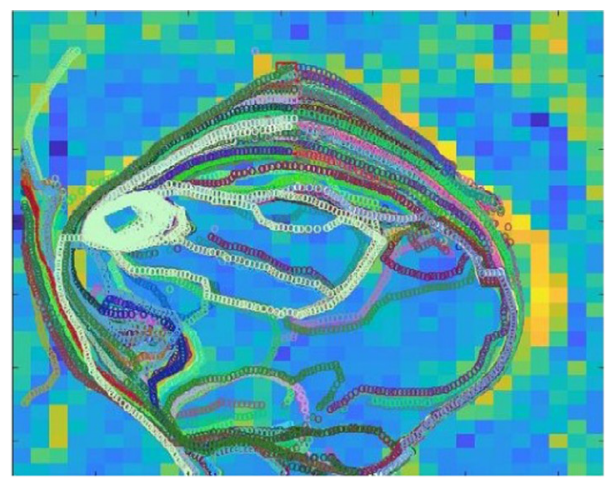

(a)

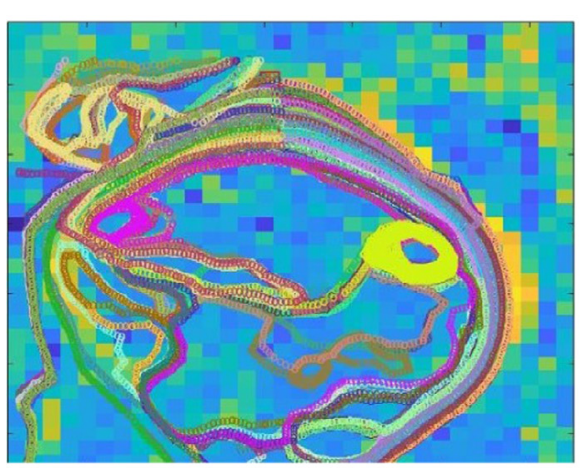

(b)

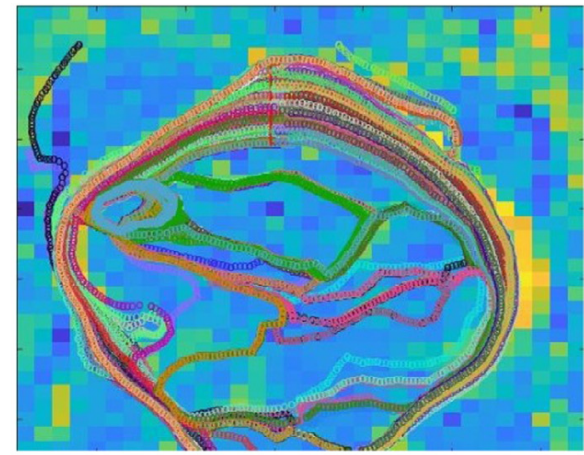

(c)

Fig. 16. Fibers computed using the hybrid approach in Real Data with (a) $D^{-1}$, (b) Adjugate metric and (c) $\beta$-scaled metric with $p=2$.

crossing fibers. These diagonal components are second order tensors lying in Riemannian symmetric space. We have shown that they have potential to effectively locate orientation distribution functions (ODFs) maxima even at small angle intersections. Our experiments showed that these diagonal components produce small orientation errors in comparison to the CT-ODF method [40] and [41], while CT-ODF was earlier shown to outperform other methods, cf. [40]. Moreover, the diagonal component method does not depend upon finding maxima of the ODFs.

In future work, we will systematically use the spectral metric approach for local interpolation. The experiment discussed in Section suggests to use spectral metric for local interpolation to preserve anisotropy which is crucial for fiber reconstruction.

In this paper, the tractography algorithms are performed using the 2nd order tensors, dealing with synthetic, phantom, and real data. We also suggest a new method how to resolve the fiber crossing, robust and efficient with small crossing angles. In near future, we plan to employ the novel fiber crossing resolution approach in global framework adapted for fiber tracking in real images.

\section{Declaration of Competing Interest}

I declare, there are no conflicts of interest of any of the coauthors related to this publication.

\section{Acknowledgments}

The first three authors have been supported by the grant MUNI/A/0885/2019 of Masaryk University, Jan Slovák gratefully acknowledges support from the Grant Agency of the Czech Republic, grant Nr. GA20-11473S.
We acknowledge the core facility MAFIL supported by the Czech-BioImaging large RI project (LM2018129 funded by MEYS CR) for their support with obtaining scientific data presented in this paper.

The second author acknowledges the support of the OP VVV funded project "CZ.02.1.01/0.0/0.0/16_019/0000765" Research Center for Informatics, CTU in Prague.

\section{References}

[1] M. Lazar, A.L. Alexander, Bootstrap white matter tractography (boot-trac), NeuroImage 24 (2) (2005) 524-532, doi:10.1016/j.neuroimage.2004.08.050.

[2] G.J. Parker, H.A. Haroon, C.A. Wheeler-Kingshott, A framework for a streamlinebased probabilistic index of connectivity (PICO) using a structural interpretation of MRI diffusion measurements, J. Magn. Reson. Imaging 18 (2) (2003) 242-254, doi:10.1002/jmri.10350.

[3] M. Schober, N. Kasenburg, A. Feragen, P. Hennig, S. Hauberg, Probabilistic shortest path tractography in DTI using Gaussian process ode solvers, in: P. Golland, N. Hata, C. Barillot, J. Hornegger, R. Howe (Eds.), Medical Image Computing and Computer-Assisted Intervention - MICCAI 2014, Springer International Publishing, Cham, 2014, pp. 265-272.

[4] P.J. Basser, S. Pajevic, C. Pierpaoli, J. Duda, A. Aldroubi, In vivo fiber tractography using DT-MRI data, Magn. Reson. Med. 44 (4) (2000) 625-632. 10.1002/1522-2594(200010)44:4<625::AID-MRM17>3.0.CO;2-O

[5] T.E. Conturo, N.F. Lori, T.S. Cull, E. Akbudak, A.Z. Snyder, J.S. Shimony, R.C. McKinstry, H. Burton, M.E. Raichle, Tracking neuronal fiber pathways in the living human brain, Proc. Natl. Acad. Sci. 96 (18) (1999) 10422-10427, doi:10.1073/ pnas.96.18.10422.

[6] M. Lazar, D.M. Weinstein, J.S. Tsuruda, K.M. Hasan, K. Arfanakis, M.E. Meyerand, B. Badie, H.A. Rowley, V. Haughton, A.A. Field, A.L. Alexander, White matter tractography using diffusion tensor deflection., Hum. Brain Mapp. 184 (2003) 306-321.

[7] S. Mori, B.J. Crain, V.P. Chacko, P.C.M. Van Zijl, Three-dimensional tracking of axonal projections in the brain by magnetic resonance imaging, Ann. Neurol. 45(2) 265-269. 10.1002/1531-8249(199902)45:2<265::AID-ANA21>3.0.CO;2-3

[8] C.-F. Westin, S. Maier, H. Mamata, A. Nabavi, F. Jolesz, R. Kikinis, Processing and visualization for diffusion tensor MRI, Med. Image Anal. 6 (2) (2002) 93-108, doi:10.1016/S1361-8415(02)00053-1.

[9] S. Mori, S. Wakana, L.M. Nagae-Poetscher, P.C.M. van Zijl, MRI atlas of human white matter, Am. J. Neuroradiol. 27 (6) (2006) 1384-1385. 
[10] C. Lenglet, R. Deriche, O. Faugeras, Inferring white matter geometry from diffusion tensor MRI: application to connectivity mapping, in: T. Pajdla, J. Matas (Eds.), Computer Vision - ECCV 2004, Springer Berlin Heidelberg, Berlin, Heidelberg, 2004, pp. 127-140.

[11] E. Prados, S. Soatto, C. Lenglet, J.-P. Pons, N. Wotawa, R. Deriche, O. Faugeras, Control theory and fast marching techniques for brain connectivity mapping, volume 1, 2006, pp. 1076-1083. 10.1109/CVPR.2006.89

[12] L. O'Donnell, S. Haker, C.-F. Westin, New approaches to estimation of white matter connectivity in diffusion tensor MRI: Elliptic PDES and geodesics in a tensor-warped space, in: T. Dohi, R. Kikinis (Eds.), Medical Image Computing and Computer-Assisted Intervention - MICCAI 2002, Springer, Berlin, Heidelberg, 2002, pp. 459-466.

[13] J. Melonakos, V. Mohan, M. Niethammer, K. Smith, M. Kubicki, A. Tannenbaum, Finsler tractography for white matter connectivity analysis of the cingulum bundle, in: N. Ayache, S. Ourselin, A. Maeder (Eds.), Medical Image Computing and Computer-Assisted Intervention - MICCAI 2007, Springer, Berlin, Heidelberg, 2007, pp. 36-43.

[14] A. Fuster, T.C.J.D. Haije, A. Tristán-Vega, B. Plantinga, C.-F. Westin, L. Florack, Adjugate diffusion tensors for geodesic tractography in white matter, J. Math. Imaging Vis. 54 (2015) 1-14.

[15] N. Sepasian, Multi-valued geodesic tractography for diffusion weighted imaging, Ph.D Thesis, Eindhoven University of Technology, 2011, ISBN 978-90-3862543-0.

[16] N. Sepasian, J.H.M. ten Thije Boonkkamp, B.M. ter Haar Romeny, A. Vilanova, Multivalued geodesic ray-tracing for computing brain connections using diffusion tensor imaging, SIAM J. Imaging Sci. 5 (2012) 483-504.

[17] N. Sepasian, A. Vilanova, L. Florack, B.M. ter Haar Romeny, A ray tracing method for geodesic based tractography in diffusion tensor images, in: Proceedings of the 2008 IEEE Computer Society Conference on Computer Vision and Pattern Recognition Workshops, 2008, pp. 1-6.

[18] X. Hao, R.T. Whitaker, P.T. Fletcher, Adaptive Riemannian metrics for improved geodesic tracking of white matter, in: G. Székely, H.K. Hahn (Eds.), Information Processing in Medical Imaging, Springer, Berlin, Heidelberg, 2011, pp. 13-24.

[19] X. Hao, K. Zygmunt, R.T. Whitaker, P.T. Fletcher, Improved segmentation of white matter tracts with adaptive Riemannian metrics, Med. Image Anal. 18 (1) (2014) 161-175, doi:10.1016/j.media.2013.10.007.

[20] A. Fuster, A. Tristan-Vega, T.D. Haije, C.-F. Westin, L. Florack, A novel Riemannian metric for geodesic tractography in DTI, in: T. Schultz, G. Nedjati-Gilani, A. Venkataraman, L. O’Donnell, E. Panagiotaki (Eds.), Computational Diffusion MRI and Brain Connectivity, Springer International Publishing, Cham, 2014, pp. 97-104.

[21] S. Kaushik, J. Slovak, Hardi segmentation via fourth order tensors and anisotropy preserving similarity measures, J. Math. Imaging Vis. 2018 (5) (2019) 12.

[22] M. Descoteaux, C. Lenglet, R. Deriche, Diffusion tensor sharpening improves white matter tractography, in: Proceedings of the Medical Imaging 2007: Image Processing, 6512, International Society for Optics and Photonics, 2007, p. 65121J.

[23] S. Jbabdi, P. Bellec, R. Toro, J. Daunizeau, M. Pélégrini-Issac, H. Benali, Accurate anisotropic fast marching for diffusion-based geodesic tractography, J. Biomed. Imaging 2008 (2008) 2.

[24] P. Thomas Fletcher, Geodesic regression and the theory of least squares on Riemannian manifolds, Int. J. Comput. Vis. 105 (2) (2013) 171-185, doi:10.1007/ s11263-012-0591-y.

[25] N. Sepasian, J.H.M. ten Thije Boonkkamp, L. Astola, M. Breeuwer, A. Fuster, Modified geodesic ray-tracing for diffusion tensor imaging, in: Proceedings of the 2016 IEEE 13th International Symposium on Biomedical Imaging (ISBI), 2016, pp. 489-493.
[26] X. Pennec, P. Fillard, N. Ayache, A Riemannian framework for tensor computing, Int. J. Comput. Vis. 66 (1) (2006) 41-66, doi:10.1007/s11263-005-3222-z.

[27] C. Lenglet, M. Rousson, R. Deriche, O. Faugeras, S. Lehericy, K. Ugurbil, A Riemannian approach to diffusion tensor images segmentation, in: G.E. Christensen, M. Sonka (Eds.), Information Processing in Medical Imaging, Springer, Berlin, Heidelberg, 2005, pp. 591-602.

[28] P.T. Fletcher, C. Lu, S. Joshi, Statistics of shape via principal geodesic analysis on lie groups, in: Proceedings of the 2003 IEEE Computer Society Conference on Computer Vision and Pattern Recognition, CVPR'03, IEEE Computer Society, Washington, DC, USA, 2003, pp. 95-101.

[29] K. Krajsek, M.I. Menzel, H. Scharr, A riemannian bayesian framework for estimating diffusion tensor images, Int. J. Comput. Vis. 120 (3) (2016) 272-299, doi:10.1007/s11263-016-0909-2.

[30] E. Prados, S. Soatto, C. Lenglet, J. Pons, N. Wotawa, R. Deriche, O. Faugeras, Control theory and fast marching techniques for brain connectivity mapping, in: Proceedings of the 2006 IEEE Computer Society Conference on Computer Vision and Pattern Recognition (CVPR'06), 1, 2006, pp. 1076-1083, doi:10.1109/ CVPR.2006.89.

[31] M. Lazar, D.M. Weinstein, J.S. Tsuruda, K.M. Hasan, K. Arfanakis, M.E. Meyerand, B. Badie, H.A. Rowley, V. Haughton, A.A. Field, A.L. Alexander, White matter tractography using diffusion tensor deflection., Hum. Brain Mapp. 184 (2003) 306-321.

[32] T. Dela Haije, N. Sepasian, A. Fuster, L. Florack, Adaptive enhancement in diffusion MRI through propagator sharpening, in: Proceedings of the Computational Diffusion MRI : MICCAI Workshop, Munich, Germany, October 9th, 2015, in: Mathematics and Visualization, Springer, Germany, 2016, pp. 131-143, doi:10.1007/978-3-319-28588-7_12.

[33] A. Collard, S. Bonnabel, C. Phillips, R. Sepulchre, Anisotropy preserving DTI processing, Int. J. Comput. Vision 107 (1) (2014) 58-74, doi:10.1007/ s11263-013-0674-4.

[34] K. Koufany, Application of Hilbert's projective metric on symmetric cones, Acta Math. Sin. Engl. Ser. 22 (2004) 1467-1472, doi:10.1007/s10114-005-0755-6.

[35] J. Jost, Riemannian Geometry and Geometric Analysis, Springer Universitat texts, Springer, 2005.

[36] D.S. Tuch, T.G. Reese, M.R. Wiegell, N. Makris, J.W. Belliveau, V.J. Wedeen, High angular resolution diffusion imaging reveals intravoxel white matter fiber heterogeneity, Magn. Reson. Med.: Offic. J. Int. Soc. Magn. Reson. Med. 48 (4) (2002) 577-582.

[37] A. Barmpoutis, M.S. Hwang, D. Howland, J.R. Forder, B.C. Vemuri, Regularized positive-definite fourth order tensor field estimation from DW-MRI, NeuroImage 45 (1) (2009) S153-S162.

[38] A. Barmpoutis, B.C. Vemuri, A unified framework for estimating diffusion tensors of any order with symmetric positive-definite constraints, in: Proceedings of the 2010 IEEE International Symposium on Biomedical Imaging: from Nano to Macro, IEEE, 2010, pp. 1385-1388.

[39] M. Jayachandra, N. Rehbein, C. Herweh, S. Heiland, Fiber tracking of human brain using fourth-order tensor and high angular resolution diffusion imaging, Magn. Reson. Med.: Offic. J. Int. Soc. Magn. Reson. Med. 60 (5) (2008) 1207-1217.

[40] A.B. Y. T. Weldeselassie, M.S. Atkins, Symmetric positive semi-denite cartesian tensor fiber orientation distributions (CT-FOD), Med. Image Anal. 16 (2012) 1121-1129.

[41] Y. T. Weldeselassie, A. Barmpoutis, M. S. Atkins, Symmetric positive semidefinite Cartesian Tensor fiber orientation distributions (CT-FOD), Medical Image Analysis, 16, 6 (2012), 1121-1129.

[42] A. Barmpoutis, B. Jian, B.C. Vemuri, Adaptive kernels for multi-fiber reconstruction, in: J.L. Prince, D.L. Pham, K.J. Myers (Eds.), Information Processing in Medical Imaging, Springer, Berlin, Heidelberg, 2009, pp. 338-349. 